\section{Case Reports in Gastroenterology}

Case Rep Gastroenterol 2018;12:116-124

DOI: 10.1159/000488192

Published online: April 13, 2018 (c) 2018 The Author(s)

Published by S. Karger AG, Basel www.karger.com/crg

This article is licensed under the Creative Commons Attribution-NonCommercial 4.0 International License (CC BY-NC) (http://www.karger.com/Services/OpenAccessLicense). Usage and distribution for commercial purposes requires written permission.

\title{
Colorectal Adenocarcinoma with an Alternative Serrated Pathway
}

\author{
Makoto Eizuka ${ }^{a}$ Keisuke Kawasaki ${ }^{b}$ Yosuke Toya $^{b}$ Risaburo Akasaka ${ }^{b}$ \\ Koki Otsuka ${ }^{c}$ Akira Sasakic Takayuki Matsumoto ${ }^{\mathrm{b}}$ Tamotsu Sugai $^{\mathrm{a}}$ \\ aDepartment of Molecular Diagnostic Pathology, School of Medicine, Iwate Medical \\ University, Morioka, Japan; ${ }^{b}$ Division of Gastroenterology, Department of Internal \\ Medicine, School of Medicine, Iwate Medical University, Morioka, Japan; 'Department of \\ Surgery, School of Medicine, Iwate Medical University, Morioka, Japan
}

\section{Keywords}

$B R A F \cdot$ Microsatellite stable phenotype $\cdot$ Immunohistochemistry $\cdot$ Sessile serrated adenoma/polyp

\begin{abstract}
In a 64-year-old woman, we identified a flat, elevated lesion that was located at the caecum and was composed of 3 different areas (areas A, B, and C). We diagnosed it as "carcinoma with sessile serrated adenoma/polyp (SSA/P)" histologically. Although area A was diagnosed as classical SSA/P, area B was regarded as a high-grade SSA/P. In contrast, area $C$ showed a differentiated-type adenocarcinoma that invaded the submucosa. The patient had a recurrence of cancer 1.5 years after endoscopic resection. Overexpression of TP53 was detected in area $C$. Although BRAF mutation was detected in all areas, CpG island methylator phenotypehigh cancer was found only in area $C$. The genomic phenotype of the cancerous tissue was classified as microsatellite stable ( $M L H 1$ gene not methylated). In the present case, we showed that a lesion with genetic alterations based on the histological sequence SSA/P $\rightarrow$ high-grade SSA/P $\rightarrow$ cancer in SSA/P and an alternative serrated pathway may exhibit aggressive behavior.

(C) 2018 The Author(s)

Published by S. Karger AG, Basel
\end{abstract}

KARGER
Tamotsu Sugai, MD

Department of Molecular Diagnostic Pathology

Iwate Medical University

19-1 Uchimaru, Morioka 020-8505 (Japan)

E-Mail tsugai@iwate-med.ac.jp 


\section{Case Reports in Gastroenterology}

Case Rep Gastroenterol 2018;12:116-124

DOI: $10.1159 / 000488192$

(c) 2018 The Author(s). Published by S. Karger AG, Basel www.karger.com/crg

Eizuka et al.: Colorectal Adenocarcinoma with an Alternative Serrated Pathway

\section{Introduction}

Sporadic colorectal cancer (CRC) can arise through 2 distinct pathways: chromosomal instability (CIN) and microsatellite instability (MSI). CIN is characterized by an alteration at the chromosomal level [1]. Similarly, the microsatellite stable (microsatellite stability; MSS) phenotype has been identified as a CIN phenotype in colorectal carcinogenesis. On the other hand, MSI is caused by DNA methylation of the promoter region of the MLH1 gene, which is one of the mismatch repair genes [2,3]. MSI is also characterized by mutation of BRAF and the $\mathrm{CpG}$ island methylator phenotype (CIMP) that is characterized by genome-wide promoter CpG island methylation in the tumor cells [3]. Sessile serrated adenoma/polyp (SSA/P) has attracted a great deal of attention as a precursor lesion of CRC with an MSI phenotype [4]. However, a recent study has shown that not only MSI but also MSS is an important target lesion in CRCs that progress through the serrated pathway.

We report a case of a 64-year-old woman with MSS CRC derived from SSA/P with an alternative pathway that is characterized by BRAF mutation and the CIMP-high phenotype during tumor progression.

\section{Case Presentation}

A 64-year-old woman with no symptoms had a positive fecal occult blood test. The findings on physical and laboratory examinations were unremarkable. Colonoscopy was performed because of the positive fecal occult blood test.

\section{Endoscopic Findings}

Colonoscopy showed a $20 \times 16 \mathrm{~mm}$ white, flat, elevated lesion with a reddish nodule in the center of the mass (Fig. 1a) located at the caecum. Chromoendoscopy with crystal violet solutions revealed mucus covering a wide-open pit (Fig. 1b) and a large dendritic structure (Fig. 1c). At the central area, the reddish nodule displayed an irregular glandular structure (Fig. 1d). These endoscopic findings were suggestive of intramucosal cancer with SSA/P. Endoscopic submucosal dissection (ESD) was performed for this tumor.

\section{Histological Findings}

Macroscopically, the resected lesion was a flat, elevated tumor, measuring $20 \times 16 \mathrm{~mm}$ in size. Upon histological examination of the resected specimen, we determined that the tumor was composed of 3 areas (areas A, B, and C) (Fig. 2a, b). Area A had crypt dilatation, irregular branching, and abnormal histological architecture at the crypt base (Fig. 2c). Area A was diagnosed as SSA/P. Area B showed more irregular branching and less abnormal architecture at the crypt base than area A (Fig. 2e). Although the cellular atypia of area B was not different from that of area A, the structural atypia of area B was greater than that of area A. The histological diagnosis of area B was high-grade SSA/P according to the structural atypia. Area $\mathrm{C}$ demonstrated cytological atypia and a complex architecture with tubular or cribriform findings (Fig. 2d). We made a histological diagnosis of well to moderately differentiated adenocarcinoma in area $\mathrm{C}$. The adenocarcinoma component invaded the submucosal layer. Lymph and blood vessel invasion was not evident. 


\section{Case Reports in Gastroenterology}

Case Rep Gastroenterol 2018;12:116-124

DOI: $10.1159 / 000488192$

(c) 2018 The Author(s). Published by S. Karger AG, Basel www.karger.com/crg

Eizuka et al.: Colorectal Adenocarcinoma with an Alternative Serrated Pathway

Immunohistochemical Findings

Immunohistochemical examination was performed with an autoimmunostaining system (Dako EnVision System, Denmark). The tumor cells in area A were positive for MUC2 (Ccp58; Novocastra Laboratories, Newcastle, UK), MUC5AC (CLH2; Novocastra Laboratories), MUC6 (CLH5; Novocastra Laboratories), CDX-2 (CDX2-88; Biogenex), MLH1 (G168728; BD Pharmingen), MSH2 (FE11; Calbiochem), MSH6 (EPR3945; Abcam), PMS2 (B-3; Santa Cruz), and Annexin A10 (polyclonal; Novus Biologicals), and they were negative for CD10 (56C56; Novocastra Laboratories) and TP53 (DO-7; Novocastra Laboratories). Although the staining pattern of area B was similar to that of area A, area B was negative for MUC6. Finally, the tumor cells in area C were positive for MUC2, MUC5AC, MLH1, MSH2, MSH6, PMS2, and Annexin A10, whereas they were negative for MUC6 and CD10. Overexpression of TP53 was seen in area C. The immunohistochemical findings are shown in Figure 3.

\section{Molecular Findings}

Tumor tissue was obtained from the 3 areas (A, B, and C) of the tumor, separately. To identify the genetic and epigenetic alternations in this tumor, we examined mutations of $K R A S$ and $B R A F$, DNA methylation, and MSI. Mutation analyses of KRAS and BRAF genes were performed with a pyrosequencing method. The cutoff value of the mutation was determined to be $>15 \%$ of tumor cells. In addition, the DNA methylation status of the gene promoter regions we examined was quantified by analysis of PCR bisulfite-modified genomic DNA (EpiTect Bisulfite Kit; Qiagen, Valencia, CA, USA) with a pyrosequencing method (PyroMark Q24; Qiagen NV), as previously described [5]. The cutoff value for DNA methylation status was $>30 \%$ of tumor cells. DNA methylation status was classified as low methylation epigenotype, intermediate methylation epigenotype, and high methylation epigenotype (HME) according to a previous report [6].

MSI status was determined by 5 NCI markers: BAT25, BAT26, D2S123, D5S346, and D17S250. MSI-high (MSI-H) was defined as $\geq 2$ markers being unstable, MSI-low was defined as 1 marker being unstable, and MSS was defined as the absence of instability. MSS-low together with MSI-low and MSS was considered to be MSS status in the present case [7].

$B R A F$ mutation (codon 600E GTG-GAG transversion) was commonly found in areas A, B, and C. Although the DNA methylation status of areas A and B was intermediate, a DNA methylation epigenotype (intermediate methylation epigenotype) was found, and that of area $C$ was a high DNA methylation epigenotype (HME). MSS was common in areas A, B, and C. Therefore, MSS, not but MSI, was detected in all areas (MSS phenotype).

A follow-up colonoscopy was performed 6 months, 1 year, and 1.5 years after ESD. However, a follow-up colonoscopy performed 1.5 years after ESD revealed local recurrence.

\section{Clinicopathological and Molecular Findings of the Resected Tumor}

Macroscopically, the resected lesion was an elevated tumor, measuring $10 \times 8 \mathrm{~mm}$ in size. Histological examination revealed a moderately and well differentiated adenocarcinoma that had invaded into the subserosa. Carcinoma had not spread to the lymph nodes.

Immunohistochemically, the tumor cells were positive for MUC2, MUC5AC, MLH1, MSH2, MSH6, PMS2, Annexin A10, and TP53, and they were negative or reduced for MUC6, 
CD10, and CDX-2. The tumor cells showed BRAF mutation (codon 600E GTG-GAG transversion), HME, and MSS.

The molecular alterations occurring in ESD and resected specimens are summarized in Table 1.

\section{Discussion}

Sporadic CRC is a heterogeneous disease in terms of pathological and molecular findings [6]. CRCs and their pathological precursors display distinct molecular signatures and distinct pathological features $[1-4,8]$. There are at least 2 major molecular pathways to CRC, including the predominant CIN pathway and sporadic MSI-H cancers that finally progress to the pure MSI pathway resulting from methylation of the promoter region of the MLH1 gene [1-4, 8]. Furthermore, the CIMP pathway, which is the other major pathway to sporadic CRC, characterizes specific lesions of sporadic CRC and the precursor lesion that is closely associated with CRC with the MSI phenotype [1-4, 8]. Although SSA/P has been thought to progress to CRC with the MSI-H phenotype so far, it is well recognized that SSA/P may progress to CRC with the MSI phenotype [2-4, 8]. However, the distinct pathological and molecular features of CRC with the MSS phenotype that arises from SSA/P have not been investigated. We report a case of CRC with the MSS phenotype derived from SSA/P.

The association of molecular markers with the histologic subtypes of benign serrated lesions and CIMP-high tumors has led to the proposal of an HP $\rightarrow \mathrm{SSA} / \mathrm{P} \rightarrow \mathrm{SSA} / \mathrm{P}$ with cytological dysplasia $\rightarrow$ cancer sequence [9]. MSI where the promoter region of the MLH1 gene is methylated is required to progress to increasing atypia (cytological dysplasia) or cancer [9]. In addition, methylation of the MLH1 gene results in negative/low expression of MLH1 and/or PMS2. In this case, SSA/P $\rightarrow$ high-grade SSA/P $\rightarrow$ cancerous area was observed in the same lesion. Therefore, we can trace the genetic sequence from SSA/P via high-grade SSA/P to a cancerous lesion. In the molecular alterations we examined, mutation of BRAF was commonly found in all areas. Although an intermediate methylation epigenotype was found in areas A and B, HME was observed only in area C. However, the promoter region of MLH1 was not methylated, and consequently, MLH1 was expressed in area B (corresponding to "cytological dysplasia") and area C (cancer area). As a result, it is presumed that MSI did not occur in these areas. In addition, although the gastric mucin phenotype that is common in SSA/P was commonly observed in all areas, TP53 overexpression was found only in area C, suggesting that TP53 overexpression plays a major role in the MSS phenotype of CRC originating from SSA/P.

Previous studies have shown that patients with CRC with MSI tumors have a better prognosis than those with MSS tumors [4]. However, recently, it has been reported that irrespective of $B R A F$ mutation, patients with MSS CRC with BRAF (+)/CIMP-high (+) showed a worse prognosis than patients with conventional CRC with the MSS phenotype [10]. In the present case, this patient relapsed in the first 18 months after ESD treatment. This finding may be supported by the finding that TP53 is overexpressed in the early phase (intramucosal cancer) of this tumor [4]. We suggest that careful observation should be taken when such patients with MSS CRC with BRAF (+)/CIMP-high (+) are found in routine pathological practice, as they may experience rapid recurrence of the primary tumor. 
In conclusion, we report a novel case of CRC with the MSS phenotype derived from SSA/P that is characterized by BRAF mutation and CIMP-high status. This tumor showed rapid recurrence after endoscopic resection. Therefore, this tumor is thought to have an aggressive behavior. In addition, in the present case, we could examine the genetic alterations of each lesion (SSA/P $\rightarrow$ high-grade SSA/P $\rightarrow$ cancer in SSA/P) within the same tumor.

\section{Acknowledgments}

We gratefully acknowledge the technical assistance of the members of the Department of Molecular Diagnostic Pathology, Iwate Medical University.

\section{Statement of Ethics}

The authors have no ethical conflicts to disclose.

\section{Disclosure Statement}

The authors declare that they have no conflicts of interest.

\section{References}

1 Lengauer C, Kinzler KW, Vogelstein B. Genetic instability in colorectal cancers. Nature. 1997 Apr;386(6625):623-7.

2 Jass JR, Whitehall VL, Young J, Leggett BA. Emerging concepts in colorectal neoplasia. Gastroenterology. 2002 Sep;123(3):862-76.

3 Ogino S, Goel A. Molecular classification and correlates in colorectal cancer. J Mol Diagn. 2008 Jan;10(1):1327.

4 Leggett B, Whitehall V. Role of the serrated pathway in colorectal cancer pathogenesis. Gastroenterology. 2010 Jun;138(6):2088-100.

5 Yamamoto E, Suzuki H, Yamano HO, Maruyama R, Nojima M, Kamimae S et al. Molecular dissection of premalignant colorectal lesions reveals early onset of the CpG island methylator phenotype. Am J Pathol. 2012 Nov;181(5):1847-61.

6 Kaneda A, Yagi K. Two groups of DNA methylation markers to classify colorectal cancer into three epigenotypes. Cancer Sci. 2011 Jan;102(1):18-24.

7 Boland CR, Thibodeau SN, Hamilton SR, Sidransky D, Eshleman JR, Burt RW et al. A National Cancer Institute Workshop on Microsatellite Instability for cancer detection and familial predisposition: development of international criteria for the determination of microsatellite instability in colorectal cancer. Cancer Res. 1998 Nov;58(22):5248-57.

8 Worthley DL, Leggett BA. Colorectal cancer: molecular features and clinical opportunities. Clin Biochem Rev. 2010 May;31(2):31-8.

9 Snover DC, Ahnen DJ, Burt RW, Odze RD. Serrated polyps of the colon and rectum and serrated ("hyperplastic") polyposis. In: Bosman FT, Carneiro F, Hruban RH, Theise ND, editors. WHO Classification of Tumours of the Digestive System, 4th ed. Lyon: World Health Organization, International Agency for Research on Cancer; 2010. p. 160-5.

10 Pai RK, Jayachandran P, Koong AC, Chang DT, Kwok S, Ma L et al. BRAF-mutated, microsatellite-stable adenocarcinoma of the proximal colon: an aggressive adenocarcinoma with poor survival, mucinous differentiation, and adverse morphologic features. Am J Surg Pathol. 2012 May;36(5):744-52. 

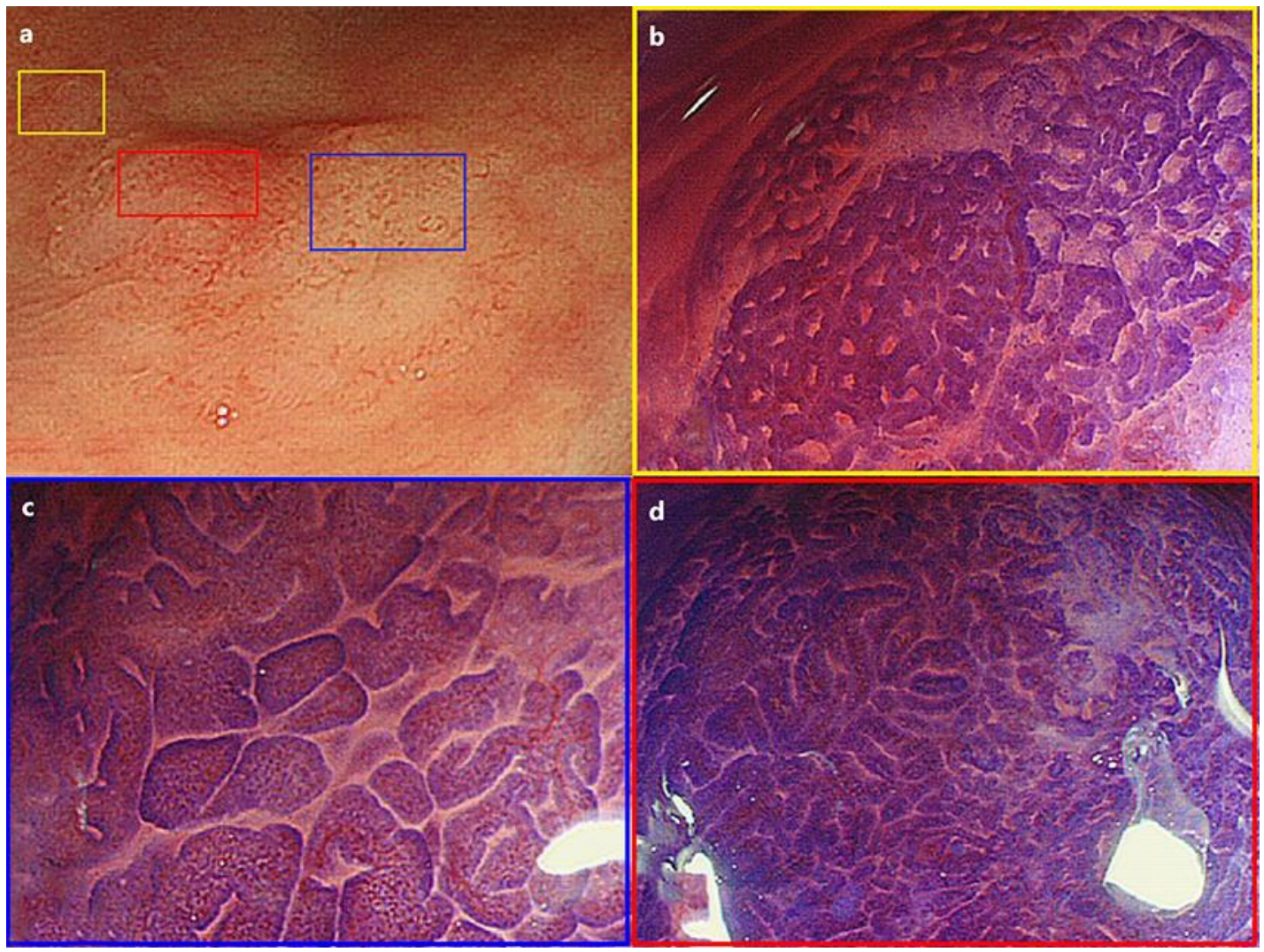

d

Fig. 1. Endoscopic features of the lesion at the caecum. a Colonoscopy detected a $20 \times 16 \mathrm{~mm}$ white, flat, elevated lesion with a reddish nodule in the center of the mass. b Chromoendoscopy of crystal violet solutions showed mucus covering a wide-open pit (square yellow box). c Crystal violet solutions revealed mucus covering a dendritic structure (square blue box). $\mathbf{d}$ The central reddish nodule demonstrated an irregular glandular structure (square red box). 
Eizuka et al.: Colorectal Adenocarcinoma with an Alternative Serrated Pathway
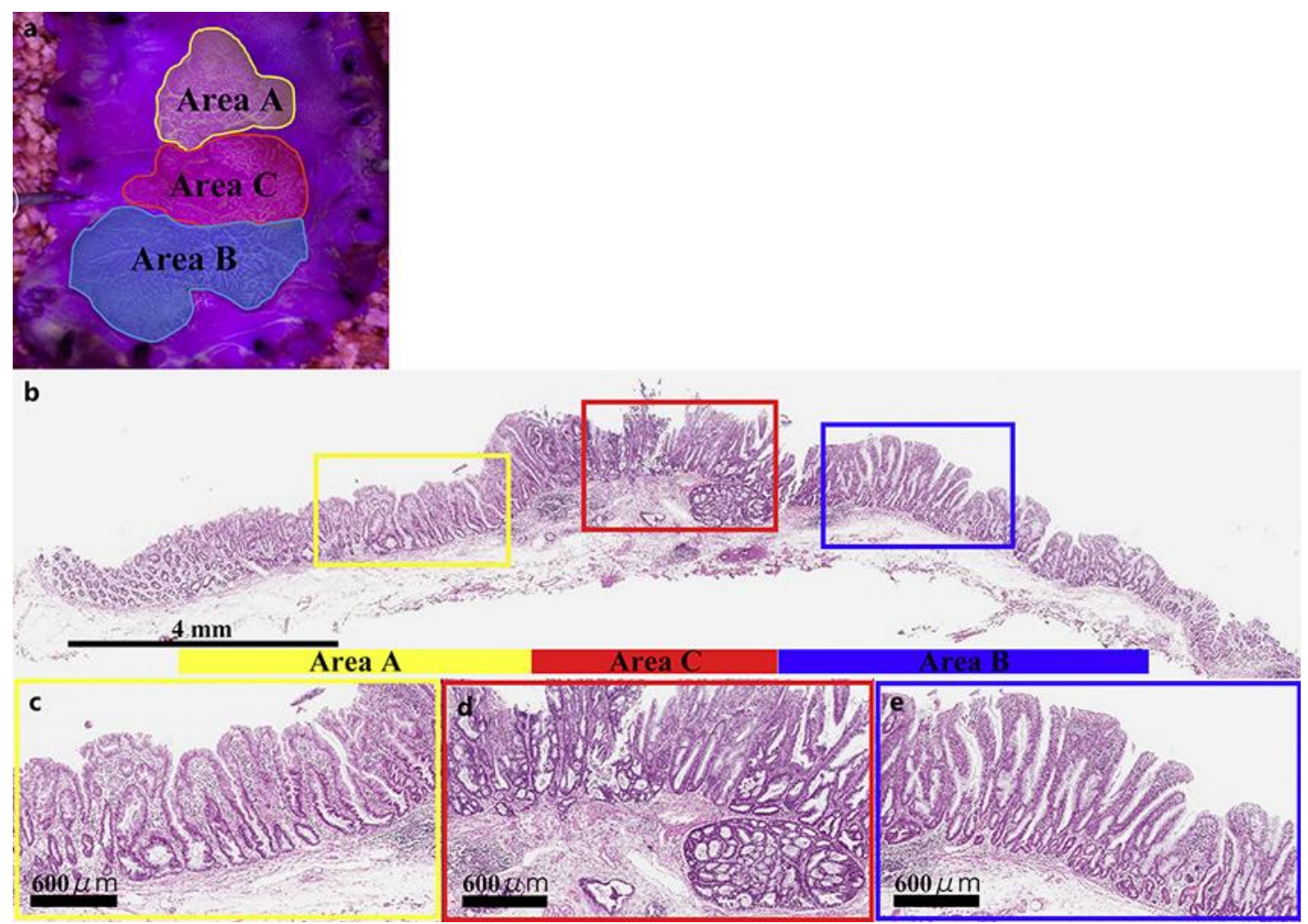

Fig. 2. Histological features of the resected tumor. a Three regions, including area A (square yellow box), B (square blue box), and C (square red box), were formed in the tumor. $\mathbf{b}$ The tumor was composed of 3 areas (areas A, B, and C) histologically. c Area A (endoscopically, a wide-open pit was detected) revealed crypt dilatation, irregular branching, and abnormal histological architecture at the crypt base, suggesting SSA/P. d Area C (endoscopically, an irregular glandular structure was detected) demonstrated cytological atypia and a complex architecture with tubular or cribriform pattern, indicating carcinoma (differentiatedtype adenocarcinoma) that invaded the submucosa (depth of submucosal invasion, 1,250 $\mu \mathrm{m}$ ). e Area B (endoscopically, a large dendritic structure was detected) showed more irregular branching and less abnormal architecture at the crypt base than area A, suggesting high-grade SSA/P. SSA/P, sessile serrated adenoma/polyp. 

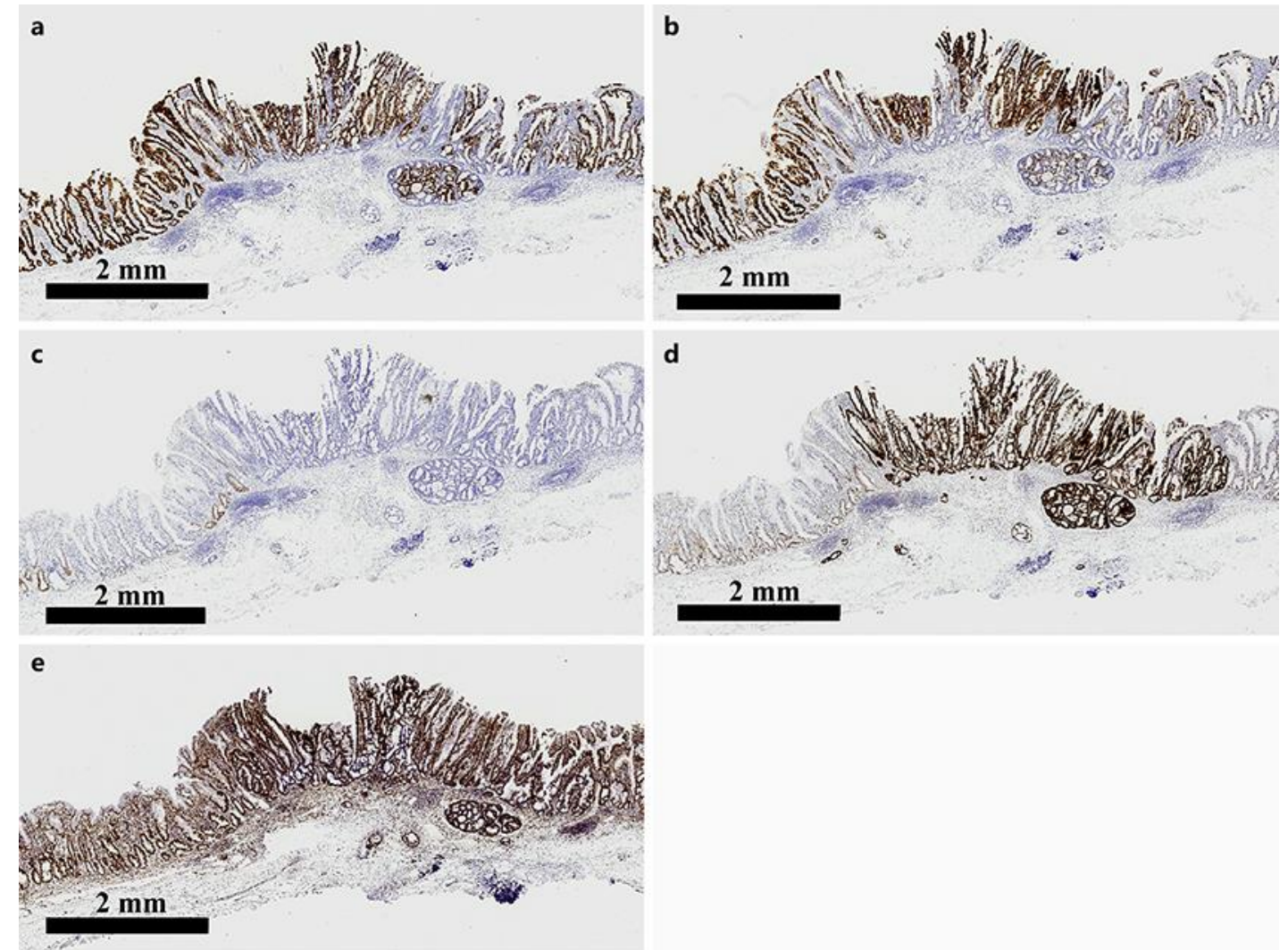

Fig. 3. Immunohistochemical findings of the tumor. a Expression of MUC2 was commonly seen in areas A, B, and C. b MUC5AC was commonly expressed in areas A, B, and C. c Expression of MUC6 was found in area A. d TP53 overexpression was seen in areas B and C. e MLH1 expression was found in areas A, B, and C. Note that MLH1 was expressed in areas B and C. 
Eizuka et al.: Colorectal Adenocarcinoma with an Alternative Serrated Pathway

Table 1. Summary of immunohistochemical staining

\begin{tabular}{|c|c|c|c|c|}
\hline & \multicolumn{3}{|c|}{ ESD specimen } & \multirow[t]{2}{*}{ Surgical specimen } \\
\hline & area A & area B & area $\mathrm{C}$ & \\
\hline MUC2 & positive & positive & positive & focally positive \\
\hline MUC5AC & positive & positive & positive & negative \\
\hline MUC6 & positive & negative & negative & negative \\
\hline CD10 & negative & negative & negative & negative \\
\hline CDX-2 & positive & positive & focally positive & focally positive \\
\hline TP53 & negative & negative & positive & positive \\
\hline Ki-67 distribution & asymmetry & diffuse & diffuse & diffuse \\
\hline Ki-67 index & $17.5 \%$ & $51.5 \%$ & $65.8 \%$ & $66.9 \%$ \\
\hline MLH1 & positive & positive & positive & positive \\
\hline MSH2 & positive & positive & positive & positive \\
\hline MSH6 & positive & positive & positive & positive \\
\hline PMS2 & positive & positive & positive & positive \\
\hline Annexin A10 & positive & positive & positive & negative \\
\hline
\end{tabular}

ESD, endoscopic submucosal dissection. 